\title{
On Development of 100-Gram-Class Spacecraft for Swarm Applications
}

Fred Y. Hadaegh; Fellow, IEEE, Soon-Jo Chung; Senior Member, IEEE, and Harish M. Manohara, Member, IEEE

\begin{abstract}
A novel space system architecture is proposed that would enable 100-gram-class spacecraft to be flown as swarms (100s to 1000s) in low Earth orbit (LEO). Swarms of Silicon Wafer Integrated Femtosatellites (SWIFT) present a paradigmshifting approach to distributed spacecraft development, missions, and applications. Potential applications of SWIFT swarms include sparse aperture arrays and distributed sensor networks. New swarm array configurations are introduced and shown to achieve the effective sparse aperture driven from optical performance metrics. A system cost analysis based on this comparison justifies deploying a large number of femtosatellites for sparse aperture applications. Moreover, this paper discusses promising guidance, control, and navigation methods for swarms of femtosatellites equipped with modest sensing and control capabilities.
\end{abstract}

\section{INTRODUCTION}

Distributed spacecraft systems can deliver a comparable or greater mission capability than monolithic spacecraft, but with significantly enhanced flexibility (adaptability, scalability, evolvability, and maintainability) and robustness (reliability, survivability, and fault-tolerance) [1]. In this paper, we introduce a new paradigm-shifting definition of distributed spacecraft technology that could enable flight of swarms of fully capable femtosatellites, as an ultimate form of realizing responsive space that can be made to rapidly react to various forms of uncertainty.

In this paper, "swarm" refers to a collection of hundreds to thousands of spacecraft, while "femtosat" implies a 100gram-class spacecraft. There has been significant interest in small spacecraft (e.g., Cubesats [2] and satellites on printed circuit boards or silicon chips [3], [4]). The SWIFT represents a 100-gram-class spacecraft capable of six degrees of freedom (6DOF) control, built by novel three-dimensional (3D) silicon wafer fabrication and integration techniques [5]. Each femtosat can be actively controlled in all 6DOF such that a desirable synergetic behavior emerges from the interactions among spacecraft and between the spacecraft and the environment (see Fig. 1). Potential applications derived from such synergetic behaviors include sparse aperture interferometers, distributed sensors for space weather monitoring, and communication relays.

Femtosat swarms would push the frontier of the existing formation flying spacecraft concepts [6], [7], [8], [9], [10] by one

F. Y. Hadaegh and H. M. Manohara are with the Jet Propulsion Laboratory, California Institute of Technology, Pasadena, CA 91109, USA (e-mail: fred.y.hadaegh@jpl.nasa.gov and harish.manohara@jpl.nasa.gov).

S.-J. Chung is with the Department of Aerospace Engineering and the Coordinated Science Laboratory, University of Illinois at Urbana-Champaign, Urbana, IL 61801, USA (e-mail: sjchung@illinois.edu).

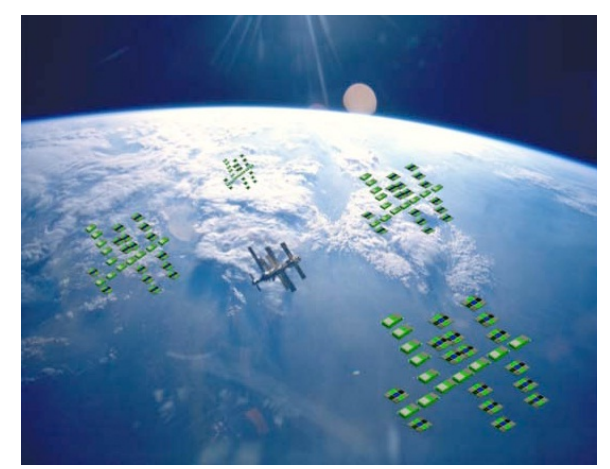

(a) SWIFT swarms mimicking the shape of the International Space Station.

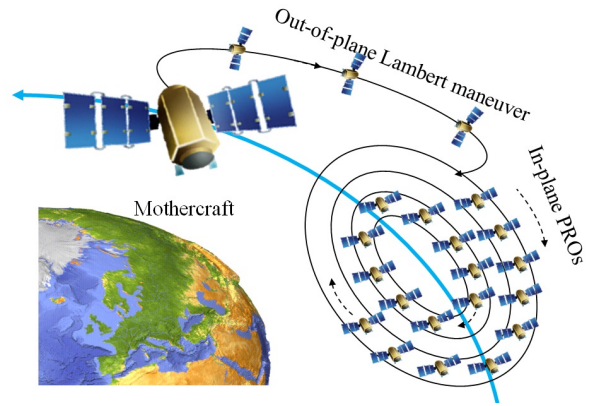

(b) Swarm deployment to passive relative orbit (PRO)

Fig. 1. A femtosat swarm is a collection of 100 gram class spacecraft designed and controlled for a desirable synergetic behavior.

or two orders of magnitude in two major technological drivers: the enormous number (1000 or more) of spacecraft, compared with the previous 2-10 spacecraft formation concepts; and a tiny size and "miniaturized" capability of 100-gram-class femtosats. As a result, the feasibility of SWIFT swarms is predicated on two enabling technological developments: (a) fabrication of 100-gram-class femtosats and (b) the individual and synergetic guidance, navigation, and control (GN\&C) capabilities of femtosats. The synergetic GN\&C capabilities of the swarm would permit coordinated maneuvers of femtosats so that the swarm can collectively exhibit or exceed a capability of more complex monolithic space systems. Furthermore, the synergetic GN\&C capabilities would drive the practicality of each swarm application and the utility of each swarm configuration. By building on recent technological advances in control, sensing, and wireless networking as well as breakthroughs in electronic packaging and fabrication, we explore fundamental system-level issues and methodologies that are unique to realizing swarm flight of femtosats. This is the essence of the swarm-centric design process introduced in this paper. 
The main contributions of this paper are as follows. First, we present generic, scalable, adaptable femtosat hardware architectures, based on a 100-gram-class wafer-scale spacecraft system including basic spacecraft functionalities and adaptable multifunctionality such as component functional redundancy. Second, we introduce several new synthetic aperture configurations and their cost analysis, which can maximize the benefit of the massively distributed spacecraft architecture. Third, we identify GN\&C strategies that are scalable and effective for a large number of spacecraft. In this paper, we challenge a conventional thinking that such controlled coordination of thousands of spacecraft is expensive and a massive amount of fuel is needed. Specifically, by studying the high-fidelity physical models of each spacecraft and deriving a collective motion of the swarm, we can indeed design a swarm of 100gram-class femtosats that are capable of forming 3-D shapes in a fuel-efficient manner.

This paper is organized as follows. In Section II, we present some key space system engineering considerations that motivate the development of SWIFT swarms, as well as some notional hardware design concepts of fabricating a 100gram-class femtosat. In Section III, we review the relative motions of spacecraft and how to exploit the dynamics to derive a fuel-efficient swarm maneuver. We also introduce GN\&C techniques that can enable formation flight of femtosat swarms. In Section IV, we introduce new random array configurations that can be used to construct sparse aperture arrays by using femtosat swarms. Based on these new swarm array configurations, a systematic cost analysis is presented.

\section{OVERVIEW OF SWIFT SWARM SySTEM}

\section{A. System Perspectives and Motivation of the Work}

The femtosat swarm architecture is motivated by the need for pushing the envelope of flexibility and robustness that can be achieved by distributed spacecraft systems. The status-quo design process always results in increased size and system complexity when faced with technological and programmatic uncertainty. In order to break this trend, we envision a flexible space architecture of a multitude of tiny spacecraft distributed over a certain shape that can be incrementally launched and replaced to cope with uncertainty in demands and technological failures. In other words, each femtosat is becoming an inexpensive "LEGO" block that can comprise a much more complicated space system. In order to articulate cost benefits of the femtosat swarm architecture, we will present a parametric cost analysis on the sparse sensing application in Section IV.

One crucial advantage of SWIFT swarms is a dramatically reduced level of risk associated with technical faults of a single spacecraft or a subset of the swarm. Because of the distributed nature of the swarm architecture, propagation of a single failure to the whole swarm can be prevented by simply retiring the faulty units from the swarm system. The two important aspects of reducing the system cost are breaking the scaling law of manufacturing a complex monolithic system, presented in Section IV, as well as cost reduction from learning curve effects [11]. The reduced spacecraft cost permits anomalous

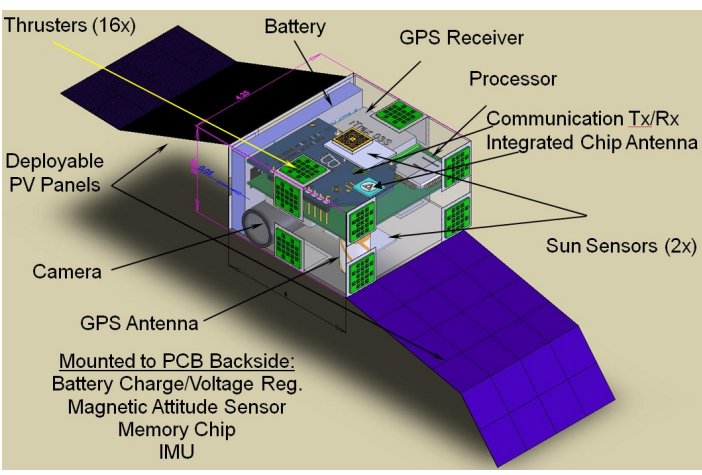

(a) Digital microthruster system (total power $=1.6 \mathrm{~W}$ and total mass $=95.5 \mathrm{~g}$ )

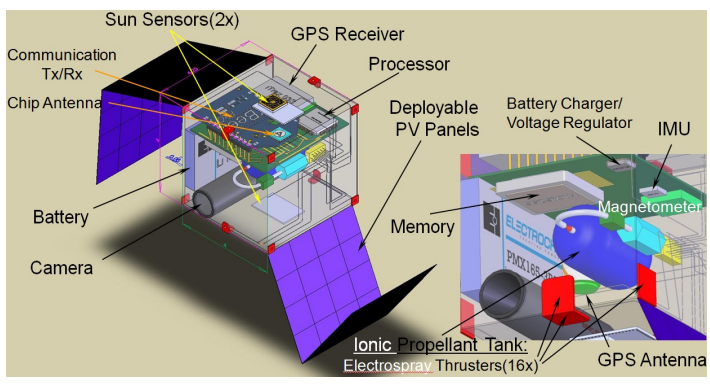

(b) Miniaturized warm gas hydrazine system (total power = $1.7 \mathrm{~W}$ and total mass $=104.7 \mathrm{~g}$ )

Fig. 2. JPL notional designs of fully capable femtosats with two different propulsion systems. The femtosat measures $4 \times 4 \times 4.25 \mathrm{~cm}$.

femtosats to be discarded and replaced gracefully without degrading the overall system performance. If the propellant usage is not uniform across femtosats in some desired swarm configurations, we can easily redistribute the femtosats so that each femtosat maintains the same level of $\Delta V^{1}$. This way, each spacecraft lifetime can be maximized. Reconfiguration of the swarm formation can also enable upgrading of the functionality of the entire swarm system whenever more advanced femtosats are available (e.g., recall Moore's law [12], especially since the SWIFT design relies on semiconductor technology).

\section{B. Baseline Functional Design of Femtosats}

Figure 2 shows two femtosat designs that use two different $6 \mathrm{DOF}$ propulsion systems. Based on the functional requirements of components shown in Table I, the development of a fully capable 100-gram-class femtosat hinges on (a) successful miniaturization of the propulsion system; (b) component multifunctionality or multichip modules; and (c) low-mass, low-power electronics for long-distance communication. It is necessary to establish a baseline of components, fabrication/packaging schemes, and the effects of local environments (thermal, radiation, drag, etc.) to understand the feasibility of building femtosats. Without such a baseline, it is difficult to develop a fully capable, functional, generic femtosat architecture. By developing a baseline and constructing a functional femtosat design, we identify technology gaps that need to be bridged to enable femtosat swarms in this section.

\footnotetext{
${ }^{1} \Delta V$ (in $\mathrm{m} / \mathrm{s}$ ) is a measure of control effort and propellant usage needed to transfer from one orbital trajectory to another.
} 
TABLE I

KEY SUBSYSTEM REQUIREMENTS OF A FEMTOSAT

\begin{tabular}{ll}
\hline Communication & within swarm $(<1 \mathrm{~km})$; groundlink $(<1,000 \mathrm{~km})$ \\
Attitude senors & accuracy $(0.1 \mathrm{deg})$ and $(<15 \mathrm{deg} / \mathrm{hr})$ for gyro \\
Position \& ranging & GPS $(<10 \mathrm{~m}[95 \%]) ; \operatorname{RFID~}(<1 \mathrm{~m}) ; \operatorname{RF}-$ based $(<10 \mathrm{~m})$ \\
Power & power generation $(4 \mathrm{~W})$ \\
Propulsion & $I_{\mathrm{sp}} \geq 100 \mathrm{~s} ; \Delta V=24 \mathrm{~m} / \mathrm{s}$ for three months \\
Command \& data & processor $(>8 \mathrm{MIPS})$ and memory $(>32 \mathrm{kB})$ \\
\hline
\end{tabular}

1) Fabrication Schemes and Component Selections: The discrete component scheme uses a breadboard approach where Commercially available off-the-shelf (COTS) or standalone custom components are assembled on a predesigned board. This approach is low-cost and highly modular albeit bulky and power hungry. On the other hand, wafer-level integration and fabrication employs a wafer-level spacecraft that is mostly monolithically fabricated. While the wafer-level integration offers a low-power, compact design, it suffers from less modularity and increased costs. Hence, we will employ chiplevel integration, which is in between the discrete component scheme and wafer-level integration where specific set of components are integrated either within a package or separate individual packages.

Chip-level integration along with an adaptation of MultiChip Module (MCM) process offers advantages of increased modularity as well as low power consumption. Also, chiplevel integration enables efficient thermal management and radiation shielding. We will explore a 3-D integration technique that is attractive from the point of decreasing the overall spacecraft size by increasing the component density. The 3D integration technique, while can be a highly customized approach, can enable sophisticated spacecraft designs because of 3-D packaging similar to Microscopic Integrated Processing Technology (MIPTEC) [13]. This can make the packaging multifunctional in that, not only does the topology serve as substrate to run solid state wiring, but can also function as a radiation shield as well as a heat dissipator. Our current effort is focusing on these two techniques to identify technology gaps. The feasibility of new femtosat designs are being explored by using the fabrication and integration techniques outlined above.

Generation of a component trade space is an iterative process. This is undertaken to first establish the baseline of available components for a 100-gram-class spacecraft. Further, this matrix of a tradespace will help identify areas of technology development in components and subsystems suitable for a femtosat. The starting criterion is to collect all possible COTS components that have been considered for small spacecraft (1 $\mathrm{kg}$ or less). Those components whose masses are greater than $100 \mathrm{~g}$ are eliminated from consideration. Components that are not space qualified can be included if their mass, functional capability, and power consumption are highly desirable for a femtosat. Such information immediately shows the technology development areas (e.g., miniature transceivers), as well as future space qualification issues.

Packaging is the glue that brings subsystems together. Generally satellites are integrated and interconnected from physically separate subsystems. Through state-of-the-art tech- nologies, electronics can be embedded in a modular fashion so as to function as a structure, passive radiator, radiation shield, instrument sensor, and/ or mechanical device, eventually resulting in a system-on-chip containing all subsystem electronics into one module.

Critical packaging factors in consideration include (a) mission life vs. disposable spacecraft attributes; (b) test and rework-ability (modularity vs. integration levels); (c) power and thermal environment (temperature and radiation); (d) instrument electromechanical integration; and (e) emerging technologies (cost) vs. mature technologies (mass). The goal is to establish a robust subsystem connectivity for chip integration. We are also considering a foldable/ flexible interconnection technology as well as multifunctional micro-electromechanical systems (MEMs) based structure technologies. The challenges to be overcome include improved reliability based on miniaturization, input/output (I/O) interfaces (signal and power in-out) capabilities, and semiconductor fabrication techniques that enable such wafer-level and chip-level integration.

2) Communication: Within the swarm, a COTS part such as the XBee Pro series $2.4 \mathrm{GHz}$ communication chip with a built-in antenna is suitable. This has a range of $1 \mathrm{~km}$ that covers the range required for swarm dimensions under consideration. For ground links, there is no lightweight/lowpower COTS communication component with a range of 700 to $900 \mathrm{~km}$ as required for ground link that can be mounted on a femtosat. Hence, this is a technology development area. Swarm configurations can be conceived that allow a ground link from a mothercraft that can house a heavier, high power communication module. This may restrict the communication to serial data transfer and swarm operation.

3) Attitude Sensors/Actuators and Power System: The most suitable attitude sensing option is a sun sensor. The JPL sun sensor [14] is conducive to monolithic integration with the femtosat body. It weighs $\sim 1$ gram with a package and consumes $\sim 25 \mathrm{~mW}$. It can be multifunctional, acting also as a range finder in a swarm through triangulation. Among COTS components there are three-axis inertial measurement units (IMUs) from Invensense [15] and Analog Devices [16] that can be used as support sensors. Star cameras based on backside-illuminated delta-doped complementary metal oxide semiconductor (CMOS) imagers are attractive both for attitude sensing as well as functioning as imaging payloads. For attitude control options, passive attitude control techniques (magnetic and hysteresis) are lightweight and require no external power source, but they are too slow to respond for some applications. Although MEMS reaction wheels are an attractive option, custom-fabricated monolithic wheels provide too little torque $\left(10^{-9}\right.$ to $\left.10^{-10} \mathrm{Nm}\right)$. However, COTS cell phone vibration motors are suitable, providing $2 \times 10^{-5} \mathrm{Nm}$.

For position sensing, COTS global positioning system (GPS) sensors are the best option. Since space qualified GPS modules are too heavy ( $\sim 30 \%$ of the femtosat mass), other civilian versions are suitable from the accuracy point of view, but require radiation hardening and space-suitable software upgrade. Hence a reliable lightweight GPS sensor is a technology development area. For a power system, COTS solar cells are the most suitable, which can generate 135 
$\mathrm{mW} / \mathrm{cm}^{2}$. Typical requirements for femtosats are in the 3 to 4 $\mathrm{W}$ range $\left(<30 \mathrm{~cm}^{2}\right)$. Li-ion rechargeable batteries operate in the temperature range identified $\left(-20\right.$ to $\left.+80{ }^{\circ} \mathrm{C}\right)$ and custom fabrication may be required to suit the femtosat form factor.

4) Propulsion: The GN\&C requirements on propulsion for a swarm operation result in higher $I_{\mathrm{sp}}$ options, where specific impulse $\left(I_{\mathrm{sp}}\right)$ indicates the efficiency of a propulsion system in terms of thrust force with respect to the amount of propellant used per unit time. Passive propulsion options such as Lorentz and solar sail techniques [3] do not offer the response speed and the control resolution required. Suitable candidates for a femtosat are: (a) electrospray thrusters using indium or ionic liquid propellants, (b) miniaturized hydrazine warm gas thrusters [17], and (c) digital microthrusters [18]. Some of these propulsion options were integrated to yield a 100-gramclass functional design of a femtosat as shown in Fig. 2. Except for digital microthrusters, all thruster options require a significant technology development, although they are highly capable options. Digital microthrusters are attractive, but are for oneshot use only with a limited lifetime. Miniature hydrazine thrusters require a cleverly designed microvalve arrangement that can make them power hungry and bulky, resulting in a technology development area. Electrospray thrusters also need a technology development in miniaturized heaters and high voltage power supplies. In essence, there exists no propulsion system option that meets all of the requirements for a complete thruster system that might consist of up to 16 thrusters. Two thrust levels are desirable with an order of magnitude range. A higher thrust level is required to position the spacecraft: $\sim 100$ $\mu \mathrm{N}$, while a lower thrust level for station keeping: $\sim 10 \mu \mathrm{N}$. The total mass of a propulsion system should be limited to 40 grams (see Table I for other requirements).

\section{Dynamics AND CONTROl OF SWARMS OF FEMTOSATELLITES}

A substantial amount of literature exists in the areas of multiagent coordination and cooperative control for robotics, ground vehicles, and formation flying spacecraft. Nevertheless, none of the existing GN\&C technologies can simultaneously address (a) such an enormous number of spacecraft in swarms; (b) relatively modest control, sensing, and communication capabilities of femtosats; and (c) the complex 6DOF motions governed by Earth's gravity field and various disturbances as well as their impact on coupled-motions or swarm behaviors. In particular, the latter distinguishes the SWIFT swarm from other robotic networks. In this section, we present the GN\&C strategies that use a wide range of spatial and temporal scales of the spacecraft swarm dynamics by quantifying their dispersion and collision rates.

\section{A. Swarm Orbital Dynamics under $J_{2}$ and Atmospheric Drag}

The stringent limitations of femtosat propulsion systems and sensor/actuators necessitate the use of the accurate set of nonlinear equations of motion for the purpose of high-fidelity modeling, simulation, and development of GN\&C algorithms. For example, dominant perturbations in low Earth orbit such as $J_{2}$ effects due to Earth's oblateness [19], [20] and atmospheric

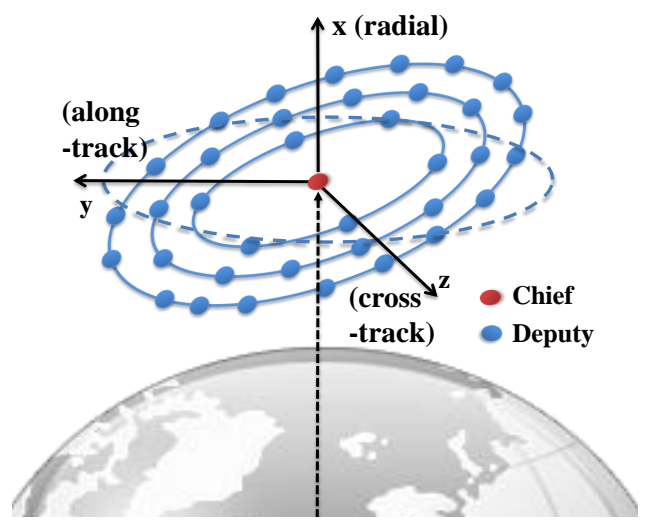

Fig. 3. PROs in the LVLH frame, which center (chief spacecraft) is orbiting the Earth in the ECI frame.

drag have to be considered to predict open-loop and closedloop swarm spacecraft motions. The Hill-Clohessy-Wiltshire (HCW) equation cannot be used for high-fidelity modeling and simulation due to its assumptions on linearization, circular orbit, and no perturbation. The high-fidelity nonlinear dynamic models for the reference (chief) and relative motions with both the $J_{2}$ perturbation and atmospheric drag were presented by either hybrid states [19] or the classical orbital parameters [21], based on [22]. We present some key attributes of swarm dynamics based on these new models.

The relative motion of a large number of deputy spacecraft (100s-1000s) on concentric PROs can be the most conveniently described in the same local vertical/local horizontal (LVLH) frame (see Fig. 3). In contrast, relative mean orbital elements [23] cannot be used for a large number of spacecraft. The origin of the LVLH frame represents either the location of a real chief spacecraft or a virtual chief orbit defined in the Earth-Centered Inertial (ECI) frame. The orbital parameters $\left(œ(t)\right.$ ) vary over time due to perturbations, notably $J_{2}$ and atmospheric drag in lower LEO (altitude $\leq 1000 \mathrm{~km}$ ), unless the motion of the chief spacecraft is perfectly controlled to be a Keplerian orbit, whose first five of the six orbital elements (semi-major axis $a$, eccentricity $e$, inclination $i$, right ascension of the ascending node $\Omega$, argument of periapsis $\omega$, true anomaly $\nu$ ) are constant. Having the fixed orbital parameters of the chief spacecraft might have negative implications for fuel usage, as discussed in Section III-E. Many of the previous models do not consider these time-varying orbital parameters in deriving the relative motions. In essence, the chief orbital motion of the chief spacecraft (or the virtual chief orbit) is derived by Gauss's variational equations [19]:

$$
\check{œ}=\mathbf{f}(œ)+\mathbf{g}(\propto) \mathbf{u}_{\text {chief }}(t)
$$

The orbital element vector $œ$ could use a hybrid representation such as $\propto=\left(r, v_{x}, h, \Omega, i, \theta\right)^{T}$ where $r, v_{x}, h$, and $\theta$ denote geocentric distance, radial velocity, angular momentum magnitude, and argument of latitude, respectively. Note $\dot{r}=v_{x}$ and $\theta=\omega+\nu$. Because of the effects of $J_{2}$ and air drag, the elements of $\mathbf{f}(\propto)$ determine the rate of change of $\propto$ while $\mathbf{g}(\propto)$ maps the 3 -axis control input $\mathbf{u}_{\text {chief into the 6- }}$ element orbital states $\propto$ if the chief spacecraft is controlled. Alternatively, see [21] for the alternative model of (1) using 
$œ=\left(a, q_{1}, q_{2}, i, \Omega, \theta\right)^{T}$ where $q_{1}=e \sin (\omega)$, and $q_{2}=$ $e \cos (\omega)$. Both representations of œ can straightforwardly be converted to the six classical orbital elements $(a, e, i, \Omega, \omega, \nu)$. By using (1), we can keep track of the time-varying orbital parameters of the chief orbit. A decision should be made as to which definition of states can reduce the computational burden of the state estimators as well as the control and guidance algorithms.

The relative motion of $j$-th spacecraft with respect to the chief orbital motion given in (1) can be derived as follows [19]:

$$
\ddot{\mathbf{x}}_{j}=\mathbf{f}_{r}\left(\mathbf{x}_{j}, \dot{\mathbf{x}}_{j}, œ, \dot{\boldsymbol{e}}, \mathbf{u}_{j}\right)
$$

where the position $\mathbf{x}_{j}=\left(x_{j}, y_{j}, z_{j}\right)^{T}$ in the LVLH frame denotes the radial, along-track, and cross-track motions as shown in Fig. 3, and $\mathbf{u}_{j}$ denotes control input (e.g., see (6)). Based on (1-2) as well as the attitude dynamics of each spacecraft, we can develop GN\&C algorithms that achieve a desired swarm configuration as discussed in Section III-D. Also, note that $œ(t)$ from (1) enters (2) as time-varying parameters, thereby indicating a hierarchical connection between (1) and (2). For example, the trajectory of the chief orbital motion $œ(t)$ needs to be known to each deputy spacecraft if its control algorithm uses (2). Any controller ignoring time-varying œ $(t)$ is regarded as a controller based on approximate models. This also leads to a state estimation problem of both œ and $\mathbf{x}_{j}$, as shall be further discussed in Section III-E. We first show results of simulation that quantify the swarm dispersion and collision rate of some useful configuration shapes that can drive the GN\&C problems.

\section{B. Swarm Geometric Configurations}

Simulation of uncontrolled motions of spacecraft $\left(\mathbf{u}_{\mathrm{chief}}=\right.$ $0, \mathbf{u}_{j}=0$ in (1-2)), initially distributed by a Gaussian distribution, results in fast dispersing unbounded motions primarily along the along-track direction (see Fig. 4). For an initial Gaussian distribution of spacecraft with $\sigma=0.5$ $\mathrm{km}$ in the $500-\mathrm{km}$ LEO orbit, the average dispersion rate is roughly $8 \mathrm{~km}$ per orbit (see Table II). This dispersion is primarily driven by the discrepancy in the energy of each orbit. In other words, each spacecraft is on a different orbit, hence with a different orbital rate, and quickly drifts with respect to one another. In such an unbounded motion with a fast divergence rate, perturbation effects such as $J_{2}$ have very little impact. On the other hand, in a slow divergence swarm configuration comprised of PROs, $J_{2}$ effects predominantly determine both the dispersion rate and the probability of collision. The acceleration due to $J_{2}$ is orders of magnitude larger than atmospheric drag (e.g., 6 times larger in 500-km orbit), and the differential orbital decay due to atmospheric drag on the relative dynamics is even smaller. Consequently, we focus on $J_{2}$ perturbations in this section and refer the readers to [19] for an analysis including atmospheric drag. First, we discuss how to construct concentric PROs.

The linearized relative motions on a circular orbit without perturbations possess a periodic solution that forms an ellipse in the projected x-y plane of LVLH (see Fig. 3). The sufficient and necessary condition for the linear relative equation to have

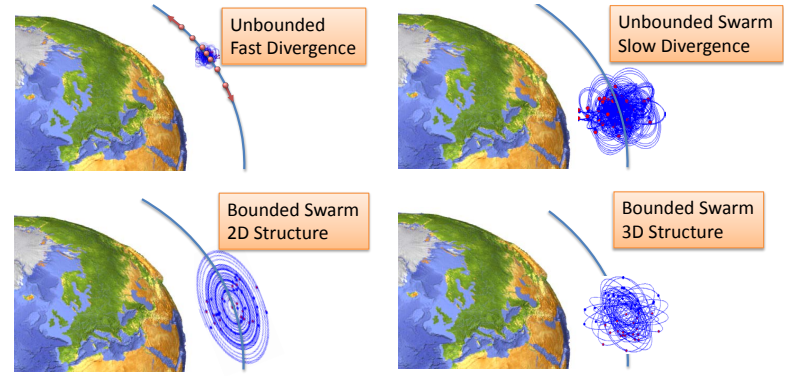

Fig. 4. Definition of swarm configurations considered in this paper.

concentric PROs is that the center of the PRO should be at the origin of the LVLH frame:

$$
\dot{y}_{\mathrm{PRO}}=-2 n x_{\mathrm{PRO}}, \quad \text { and } \dot{x}_{\mathrm{PRO}}=n y_{\mathrm{PRO}} / 2
$$

where the first condition results in a bounded elliptical orbit with the orbital rate $n$ in the LVLH frame.

This is a special case of matching the period (orbital rate) of multiple spacecraft in close proximity on a circular orbit. As shown in Fig. 3, the semi-major axis of the projected ellipse in the $\mathrm{x}-\mathrm{y}$ plane is defined along the $\mathrm{y}$-axis and twice larger than the semi-minor axis. If the first condition is not met $\left(x_{\mathrm{PRO}} \neq\right.$ 0 ), we can approximate the drift rate along the along-track direction from the HCW equation: $y(t)=-1.5 n\left(x_{\mathrm{PRO}}\right) t$. The second condition, which forces the y-center of the PRO to zero, is essential in reducing the collision rate of the swarm significantly. By using (3), we can find impulsive $\Delta V$ burns, $\dot{x}_{j}$ and $\dot{y}_{j}$ in terms of the current position $x_{j}$ and $y_{j}$. Since (3) works only under the assumption of the linear HCW equations, a complete generalization of constructing concentric ellipses by using the exact nonlinear dynamic model in the presence of $J_{2}$ is presented in [19].

The four initial states of $x_{j}, y_{j}, \dot{x}_{j}, \dot{y}_{j}$ in the LVLH frame determine the four in-plane PRO elements such as the semimajor axis of the projected PRO in the $x-y$ plane, the center location of the PRO ( $x_{\mathrm{PRO}}$ and $\left.y_{\mathrm{PRO}}\right)$, and the current phase angle $\phi_{j}$ of the projected PRO [24] (see Fig. 3). The zdirectional out-of-plane motion is independent of the $x-y$ inplane motion if the linear $\mathrm{HCW}$ equation were to be correct. The additional initial conditions of $z_{j}$ and $\dot{z}_{j}$ determine the amplitude $\left(z_{\max }\right)$ and the phase angle of the z-directional motion, thereby completing the definition of a 3-D PRO in the LVLH frame from (3). However, as pointed out in [19], in the presence of $J_{2}$ perturbations, the relative motions become increasingly coupled in all three dimensions and the $J_{2}$ invariant conditions given in (5) should be used in lieu of (3), as discussed in the next section.

A collection of multiple concentric PROs with variations of PRO semi-major axes and local tip/tilt angles can be defined and simulated under no control actuation (see Fig. 4). The dispersion rate of PROs in 500-km LEO is driven predominantly by $J_{2}$ effects and smaller than the fast divergence case by two orders of magnitude (see Table II). Hence, an uncontrolled configuration comprised of multiple concentric PROs is defined as an unbounded slow divergence swarm configuration. The swarm constructed by multiple concentric PROs retains the shape of an ellipse centered at the origin but 
TABLE II

AVERAGE SWARM CHARACTERISTICS IN LEO (500 KM, I $=45$ DEG) STARTING FROM GAUSSIAN RANDOM POSITION WITH $\sigma=0.5$ KM, 500 SPACECRAFT

\begin{tabular}{l|llll}
\hline & $\begin{array}{l}\text { Unbounded Swarm } \\
\text { Fast Divergence }\end{array}$ & $\begin{array}{l}\text { Unbounded Swarm } \\
\text { Slow Divergence }\end{array}$ & $\begin{array}{l}J_{2} \text { Invariance Swarm [19] } \\
\text { Slowest Divergence }\end{array}$ & $\begin{array}{l}\text { Bounded Swarm } \\
\text { No Divergence }\end{array}$ \\
\hline \hline Actuation & None & $\Delta V$ burns in $\mathrm{x}$ y & $\Delta V$ burns in $\mathrm{x}, \mathrm{y}, \& \mathrm{z}$ & Feedback Control \\
\hline Average $\Delta V$ & 0 & $1.1 \mathrm{~m} / \mathrm{s}$ initial & $1.5 \mathrm{~m} / \mathrm{s}$ initial & $\begin{array}{l}1.2-1.7 \mathrm{~m} / \mathrm{s} \mathrm{initial} \\
+0.02 \mathrm{~m} / \mathrm{s} / \mathrm{orbit}\end{array}$ \\
\hline Dispersion Rate & $8,000 \mathrm{~m} /$ orbit & $20 \mathrm{~m} /$ orbit & $7.6 \mathrm{~mm} / \mathrm{orbit}$ & 0 \\
\hline Collision Rate & 0 & $\begin{array}{l}5 \% \text { at } 60 \text { orbits } \\
\text { and } 78 \% \text { at } 500 \text { orbits }\end{array}$ & $<2 \%$ at 500 orbits & 0 \\
\hline
\end{tabular}

the maximum semi-major axis at each orbit (defined in the along-track direction) increases resulting in a more eccentric ellipse. The oscillatory nature of the average drift rate of the swarm distributed on concentric PROs indicates that some spacecraft are drifting towards the chief before the 70th orbit, thereby shrinking and expanding in a repeating cycle [19]. We define this behavior as a swarm breathing motion.

This result explains why swarms of spacecraft in concentric PROs exhibit much improved collision characteristics, as shown in Fig. 5a. If the distance between each spacecraft is less than $1 \mathrm{~m}$, we assume that a collision occurred. For 500 spacecraft initially distributed with various $\sigma$ values of the Gaussian distribution, we can compare the collision rates (fraction of collided spacecraft per orbit) between concentric PROs that are constructed by (3) and nonconcentric PROs. We can find that the concentric PRO configuration can considerably delay collision. However, such a collision characteristic under $J_{2}$ perturbations can be unsatisfactory. In the next section, we describe some new results of $J_{2}$-invariant swarms that can provide collision-free PROs for hundreds of orbits.

\section{C. $J_{2}$-Invariant PROs and Bounded Swarm}

One important result is construction of $J_{2}$-invariant PROs in LEO. In contrast with prior work [20], [23], [25], which uses mean orbital elements for constructing $J_{2}$-invariant chief orbits, a large number of spacecraft on multiple $J_{2}$-invariant concentric PROs can be considered only by using the relative dynamics described with respect to the single chief motion of the LVLH frame, given in (1-2). We refer the readers to Morgan et al. [19] for details. In essence, we can inflate the orbital rate $n$ of (3), thereby matching the energy of each $j$-th orbit with $J_{2}$ effects as follows.

$$
\frac{\left\|\mathbf{V}_{j}\right\|^{2}}{2}+U_{j}=\frac{\left\|\mathbf{V}_{\text {chief }}\right\|^{2}}{2}+U_{\text {chief }}
$$

where the vector $\mathbf{V}$ with each subscript indicates the velocity vector in the inertial frame and $U$ indicates the potential energy with $J_{2}$ effects given in [19].

The $J_{2}$-invariant periodic relative orbit is determined for any initial position in the LVLH frame, $\mathrm{x}_{\mathrm{PRO}}=$ $\left(x_{\mathrm{PRO}}, y_{\mathrm{PRO}}, z_{\mathrm{PRO}}\right)^{T}$, by applying the energy-matching method (4) after correcting for the directional change of the gravity gradient vector caused by the $J_{2}$ disturbance, and $J_{2}$ perturbed cross-track motions. This results in many deputies that are energy-matched as though there were no $J_{2}$, similar to the PRO solution of the HCW equation (3) as follows.

$$
\dot{\mathbf{x}}_{\mathrm{PRO}}=\mathcal{Y}\left(\mathbf{x}_{\mathrm{PRO}}, \propto\right)
$$

where the exact definition of the function $\mathcal{Y}$ can be found in [19]. We can achieve substantially fewer collisions and less drift by using (5) as shown in Fig. 5b and Table II while the fuel usage is comparable to the linear period-matched swarm by using (3). Further, a multiple-burn guidance method [19] turns out to be very effective in preventing collisions for hundreds of orbits under both $J_{2}$ and atmospheric drag perturbations. The proposed $J_{2}$-invariant PROs constructed by nonlinear energy matching (5) yield a drift rate of $7.6 \mathrm{~mm}$ per orbit and no collision for more than 500 orbits if they are reasonably separated at time zero. If the desired trajectory of each controlled femtosat is defined by a concentric PRO, then the controlled configuration is called a bounded swarm. If each concentric PRO has different tip-tilt angles, then basically a group of multiple concentric PROs form a 3-D ellipsoid shape. However, if each concentric PRO has the same tiptilt angle, then the configuration resembling a disk is called a 2-D structured shape, as shown in Fig. 4. As shown in Table II, it takes about $\Delta V$ of $0.02 \mathrm{~m} / \mathrm{s}$ per orbit to maintain a bounded swarm structure defined by multiple concentric PROs. It is found that a higher level of $\Delta V$ is required for tilted PROs than in-plane PROs $\left(z_{\max }=0\right)$. However, it takes more propellant to drive the swarm to structured configurations (e.g., 2-D disks) than just to slow dispersion by (5). It is intuitive to think that an elliptical chief orbit requires much larger $\Delta V$ since PROs are derived on the assumption of a circular orbit. Also, in LEO, the inclination of $i=45 \mathrm{deg}$ of the chief orbit yielded a smaller requirement on $\Delta V$ than a circular orbit with $i=0 \mathrm{deg}$. Optimizing concentric fuel-efficient PROs on highly elliptical chief orbits in the presence of $J_{2}$ and other disturbances is an important area of research.

\section{Guidance and Control Challenges of Swarm Flight}

The success of coordination of femtosat swarm flight hinges on the efficient GN\&C algorithms that are scalable to a very large number of femtosats. Efficient algorithms here connote both computational efficiency and optimal propellant usage. For a specific swarm application and mission scenario, the guidance and control tasks of swarms of femtosats can be divided into initial swarm deployment and distribution, swarm behavior specification and planing, swarm keeping or containment, swarm reconfiguration or resizing, passive or 


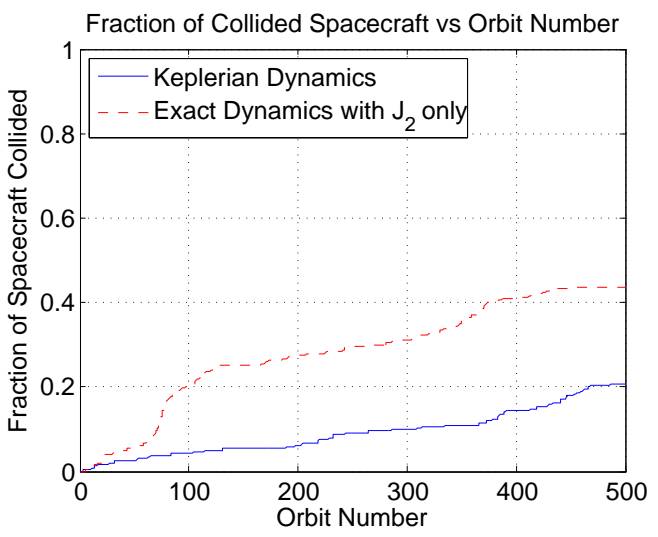

(a) Collision rates of concentric PROs from the linear $\mathrm{HCW}$ equations.

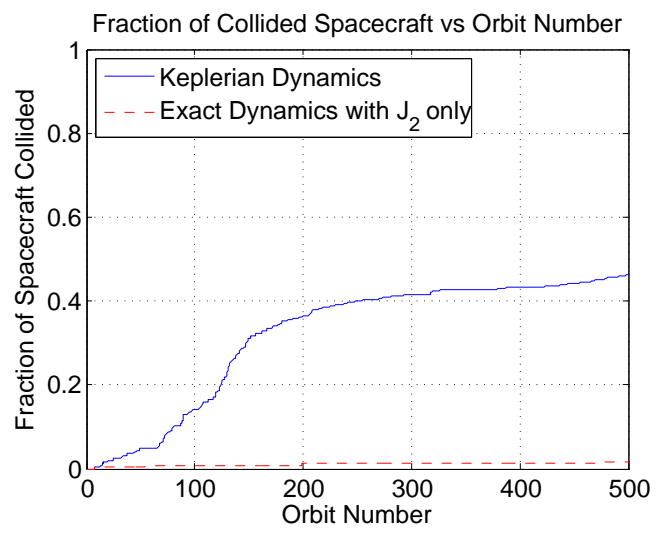

(b) Collision rates of $J_{2}$-invariant PROs from the exact nonlinear equations.

Fig. 5. Fraction of collided spacecraft with 500 spacecraft initially under a Gaussian distribution of $\sigma=0.5 \mathrm{~km}$. The chief orbit is a circular orbit with $a=500 \mathrm{~km}$ and $i=45 \mathrm{deg}$. The plots were prepared by D. Morgan.

active collision avoidance, and fault detection isolation and recovery (FDIR). For each task, the efficient guidance and control algorithms are responsible for control, coordination, and trajectory planning of femtosats by using either centralized or decentralized strategies. In this section, we present possible solutions to critical challenges posed by a large number of femtosats (100s-1000s) operating in LEO.

1) Fuel Efficient Algorithms with Highly Nonlinear Dynamics: One challenge unique to the spacecraft swarm is to meet the optimal and robust performance requirement of the desired swarm behaviors governed by both the highly nonlinear orbital dynamics and the attitude dynamics. The relatively modest control, sensing, communication, and computation capabilities of femtosats will further complicate the complexity of the GN\&C problems.

Prior work on consensus, multivehicle control, and flocking problems using graphs, particularly popular in the areas of robotics and multiagent system control [26], [27], tends to assume very simple dynamics such as linear systems and single or double integrator dynamic models. Such work cannot be directly applied to the guidance and control problem of femtosat swarms. One key difference is the complexity of the dynamic models of spacecraft swarm dynamics, as discussed in the previous sections. The algorithms developed for simple planar motions of mobile robots or aerial vehicles

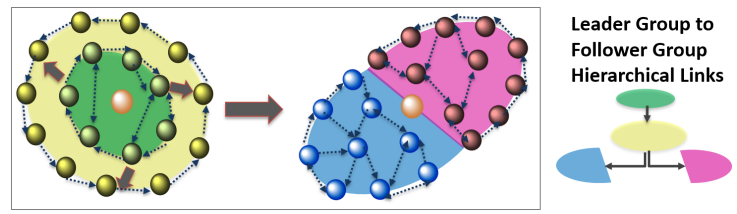

Fig. 6. Concurrent synchronization of multiple hierarchically combined groups [34]. The synchronization of the green group sends the synchronous desired input to the yellow group, whose synchronous trajectory in turn enters the red and blue groups.

can automatically ensure neither fuel-efficient nor collisionfree maneuvers for the swarm dynamics in the presence of various orbital perturbations. In other words, when we derive the GN\&C algorithms and conduct verification and validation $(\mathrm{V} \& \mathrm{~V})$, we should consider the highly nonlinear coupled timevarying dynamics with various environmental, sensor, actuator, and communication uncertainties.

The attitude control of a rigid body model of the fully 6DOF capable femtosat is also important especially for the synthetic aperture application considered in this paper. A unified synchronization control framework of highly nonlinear attitude dynamic models and relative orbital motions can be employed [28], [29]. A capability of synchronized rotation [30], [29] can integrate attitude control with control of the orbital dynamics on concentric PROs. Also, due to the modest actuation capability of a femtosat, control of coupled underactuated spacecraft [31], [32], [33] can be exploited.

2) Decentralized Feedback Control and Synchronization: Fully centralized GN\&C algorithms for a large swarm of femtosats results in significant computation and communication requirements. Consequently, decentralized algorithms should be considered while their performance and robustness issues should be verified in comparison with fully decentralized approaches. The decentralized guidance and control approaches provide several advantages that include (a) scalability and algorithmic simplicity, (b) higher accuracy and efficiency with a larger number of femtosats, (c) robustness to individual spacecraft failures, and (d) minimal onboard computational requirements.

Because of the dimensionality of femtosat swarms, it is important to ensure the scalability of modeling, guidance, and control methods. A decentralized technique is not a complete answer to this problem since there are numerous ways of pairing the femtosats with their neighbors. We have to cope with the complexity of the information-flow network structures even after decentralization. For example, the network of information flows among agents should be either on a connected undirected graph or a strongly connected directed graph (or balanced for average consensus) for consensus stability [28]. However, in a large time-varying network, it would be very restrictive to maintain such a topological requirement especially when the spacecraft swarms go through numerous reconfigurations.

One strategy to overcome such complex large-scale networks is to exploit hierarchical synchronization of multiple heterogeneous groups with multiple leaders such that two different types of coupling, such as diffusive coupling with neighbors and leader-follower couplings, are simultaneously employed [28] (see Fig. 6). This can be a generalization of the leader-follower architecture for a smaller number of 
spacecraft [35] and typical consensus (diffusive) network couplings [29], [36]. In contrast with other multiagent systems, the femtosat swarm architecture may necessitate different types of leaders. The chief spacecraft defines the origin of the local LVLH frame for a group of femtosats on the same reference (chief) orbit $\propto(t)$ as shown in Fig. 3. On the other hand, the leader spacecraft, which is different from the chief spacecraft, defines the desired trajectory that other femtosats can follow. Concurrent synchronization [37], [28] is defined as a regime where the ensemble of dynamical elements is divided into multiple groups of fully synchronized elements, but elements from different groups are not necessarily synchronized and can exhibit entirely different dynamics. By combining leaderfollower connections and local neighbor couplings, the networks are neither strongly connected nor balanced due to the reference input couplings. For example, the concurrent synchronization controller for the $j$-th femtosat dynamic model in (2) with respect to the chief motion $\propto(t)$ governed by (1) can be considered for tracking a desired trajectory defined by a physical or virtual leader spacecraft, $\mathbf{x}_{\text {leader }}$ (see [34] for the details):

$$
\mathbf{u}_{j}=\mathbf{a}_{c}\left(\mathbf{x}_{j}, \dot{\mathbf{x}}_{j}, \propto, \mathbf{s}_{j}, t\right)-\sum_{k \in \mathcal{N}_{j}}\left[\mathbf{c}_{j}\left(\mathbf{s}_{j}, t\right)-\mathbf{R}\left(\phi_{j k}\right) \mathbf{c}_{k}\left(\mathbf{s}_{k}, t\right)\right]
$$

where $\mathcal{N}_{j}$ denotes the set of neighboring femtosats, and $\mathbf{s}_{j}=\left(\dot{\mathbf{x}}_{j}-\mathbf{R}\left(\phi_{j l}\right) \dot{\mathbf{x}}_{\text {leader }}\right)+\lambda\left(\mathbf{x}_{j}-\mathbf{R}\left(\phi_{j l}\right) \mathbf{x}_{\text {leader }}\right)$ with a positive gain $\lambda$. This is a generalization of a linear consensus control law of the form $\left(\mathbf{s}_{j}-\mathbf{s}_{k}\right)$ by using both the nonlinear feedback function $\mathbf{a}_{c}(\cdot)$ and the nonlinear coupling function $\mathbf{c}_{j}(\cdot)$ along with a phase rotation $\mathbf{R}\left(\phi_{j k}\right)$ between the $j$ th and $k$ th femtosats on the periodic relative orbits. A phase-rotating transformation function can be found for $J_{2}$ invariant elliptical orbits in 3-D by setting $\mathbf{x}_{\mathrm{PRO}}=\mathrm{x}_{\text {leader }}$ in (5) [34]. A phase synchronization technique for spacecraft was first introduced in [29], [34], and more generally for artificial neural oscillators in [38], [39]. In contrast with a completely uncoupled controller for each femtosat (e.g., (6) without the coupling term $\left.\mathbf{R}\left(\phi_{j k}\right) \mathbf{c}_{k}\left(\mathbf{s}_{k}, t\right)\right)$, the concurrent synchronization controller in (6) would enhance the reconfigurability of the network, by commanding a selected set of leader spacecraft and having other members of the femtosats follow the leaders. Furthermore, in the presence of external disturbances, the synchronization control is shown to yield smaller synchronization errors than tracking errors [34]; i.e.,

$$
\lim _{t \rightarrow \infty}\left\|\mathbf{x}_{j}-\mathcal{E}\left(\mathbf{x}_{k}, \phi_{j k}\right)\right\|<\lim _{t \rightarrow \infty}\left\|\mathbf{x}_{j}-\mathcal{E}\left(\mathbf{x}_{\text {leader }}, \phi_{j l}\right)\right\| \leq \Delta
$$

where $\exists \Delta>0$ and $\mathcal{E}\left(\mathbf{x}_{k}, \phi_{j k}\right)$ rotates the position of a neighbor $\left(\mathrm{x}_{k}\right)$ by the angle $\phi_{j k}$. In other words, maintaining a formation shape takes precedence over following the desired trajectory of a leader motion in the sense of (7). See [34] for rigorous proofs of (7) and establishing connection between nonlinear stability tools for networked systems such as contraction-based incremental stability, passivity, input-tostate stability (ISS), and finite-gain $\mathcal{L}_{p}$ stability.

Another strategy to deal with network complexity is to use adaptive graph Laplacians [34], [21] that can be varied by an

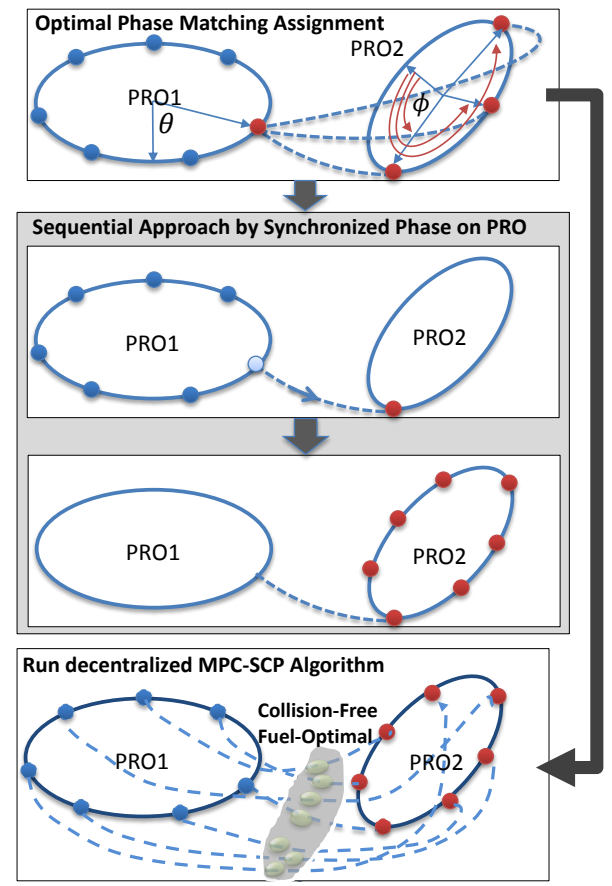

Fig. 7. Optimal collision-free reconfiguration of swarms of spacecraft on PROs by sequential and parallel approaches.

adaptive control law for the coupling gains in $\mathbf{c}_{j}$ and $\mathbf{c}_{k}$ of (6) based on the relative distances and synchronization errors. A time-varying and switching network topology, constructed by the adaptive graph Laplacian matrix, relaxes the standard requirement of consensus stability, even permitting exponential stabilization on an unbalanced digraph or a weakly connected digraph that can sporadically lose connectivity.

3) Decentralized Optimal Guidance and Probabilistic Swarm Guidance: One intriguing possibility for the femtosat swarm architecture is that we can develop and implement real-time optimal guidance and control strategies by using powerful onboard computation of the mothercraft or leader spacecraft orbiting near the femtosats. While deploying multiple computation leaders is possible, it is also feasible to put a powerful processor on each femtosat leveraging the rapid advances in the semiconductor technology (cf. multi-core processors used for smart phones). As the number of agents greatly increases, an optimal control and path planning strategy should be decentralized to remain a tractable problem. Popular optimization-based methods for real-time, multivehicle guidance include sequential convex programming (SCP) [40], [41], linear programming [27], and mixed integer linear program (MILP) [42], [43], which can also be formulated as model predictive control (MPC). In particular, a decentralized MPCSCP algorithm [41] has been derived that provide optimal, collision-free motions for thousands of spacecraft transferring between multiple PROs. Decentralization in real-time guidance can be realized by using decentralized onboard computation, intersatellite communication, and relative sensing. The disposability of mass-produced femtosats permits us to implement more advanced GN\&C algorithms that would otherwise not survive the expensive $V \& V$ process for a large space system.

It is clear that (6), which uses (5) as $\mathbf{x}_{\text {leader }}$ of a desired PRO, establishes a robust coupling feedback controller for 
tracking and stabilizing around the target $J_{2}$-invariant PROs. If the femtosats have to follow collision-free reconfiguration trajectories connecting between the initial and target PROs, we can exploit the collision-free characteristics of phased-synchronized spacecraft following $J_{2}$-invariant concentric PROs by using (6), thereby driving each femtosat to a target PRO in a sequential manner by selecting only one departing location in the initial PRO, as illustrated in Fig. 7. On the other hand, in order to compute multiple collisionfree, fuel-optimal trajectories for simultaneous reconfiguration maneuvers between multiple pairs of initial and target PROs, we can first find an optimal mapping function that finds the optimal target location $\phi$ in the target PRO for each initial location $(\theta)$ in the initial PRO (see Fig. 7). Here we make use of the synchronization controller given in the form of (6) [34] that allows us to prescribe the location of each spacecraft on the PRO by using a phase angle (e.g., $\theta, \phi \in[0,2 \pi)$ ). After we find an optimal mapping or assignment function $\phi=\mathcal{F}(\theta)$, we can solve a decentralized optimal guidance problem using those pairs of initial/terminal constraints to compute desired trajectories connecting between the initial and target PROs. The optimal guidance algorithm for a large number of spacecraft should be decentralized to reduce the computation and communication requirements [40]. The decentralized MPC-SCP algorithm [41]) has been successfully applied to the optimal guidance problem of reconfiguring hundreds of spacecraft using the high-fidelity dynamics in (12).

Another novel idea of handling a large number of femtosats in the swarm is to employ a probabilistic approach of designing a Markov chain that allows each spacecraft to determine its own trajectory in a statistically independent manner. One benefit of probabilistic swarm guidance (PSG) [5], [44] is that the swarm converges to the desired formation with an ability to automatically repair the formation if it is damaged. Recently, an inhomogeneous Markov chain approach to PSG has been proposed in order to minimize the number of transitions required for achieving the desired formation shape and to account for necessary motion constraints [44]. By using a consensus protocol, each spacecraft can estimate the overall swarm distribution to ensure desired characteristics of the inhomogeneous Markov chain.

\section{E. Navigation and Estimation for Swarm Flight}

The estimation problem for the swarm spacecraft dynamics given in (1-2) presents three main challenges: (a) scalability to a large network, (b) integrated intersatellite communication and relative sensing, and (c) nonlinearity of the dynamics and measurement equations. The control methods suggested in Section III-D can be further developed to deal with the issue of scalability and dimensionality of the estimation problem as well. The estimation algorithms for the chief orbit (œ) and relative states $\left(\mathbf{x}_{j}\right)$ can be formulated for each femtosat by using (1-2). Depending on the guidance and control algorithms, each femtosat may need to estimate the states of neighboring femtosats (e.g., the synchronization control law needs estimates of neighbors). How to define coordinate frames for both inertial and relative states will affect computational burden and accuracy of the estimation algorithms given a sensor suite available for each femtosat. The accuracy of state information is critical in computing the desired control inputs (e.g., for impulsive controls in (3) or (5) and closed-loop control in (6)).

For example, notice in (1) that the state information of the chief orbital parameters $(œ)$ is required in order for each spacecraft in the swarm to accurately estimate its own relative states $\left(\mathbf{x}_{j}\right)$. The easiest method is to assume that $\propto(t)$ follows a Keplerian orbit, which is inaccurate in the presence of disturbances such as $J_{2}$ and atmospheric drag. Then, relative state estimation with respect to this inaccurately frozen chief orbit would incur more fuel usage for femtosats to fight perturbations. Consequently, each femtosat should propagate the exact chief orbit dynamics (1) by onboard computation, thereby reducing fuel usage since femtosats need not fire thrusters to fight the $J_{2}$ drift of the chief orbit. Such a numerical integration process corresponds to a propagation step for estimation of the chief orbit. A crosslink or relative sensing between the chief spacecraft and the deputy spacecraft can form a measurement model to complete the estimator design for the chief orbit $\propto(t)$. The mothercraft or chief spacecraft can house a high-power communication module that can be used to broadcast the chief (reference) orbital parameters to swarm femtosats. Alternatively, as discussed in Section III-D, we can envision having multiple leader spacecraft that are more enhanced in terms of computational power and communication range. The leader or chief spacecraft communicate either their GPS pseudorange measurements or filtered coordinate states $\propto(t)$ and may rely on some intermediate communication relays for a large-scale swarm. The challenge is that each spacecraft needs to know the common value of $œ(t)$ at each time-step despite potential communication errors and delays. A separate consensus-based estimator [45], [46] can simultaneously estimate the common state of the leader spacecraft to reduce the discrepancy among the oe $(t)$ estimates. In addition, decentralized observers can estimate their own relative position and that of a neighboring femtosat in the common LVLH frame by using (2). Most distributed estimation algorithms [47], [45] are predicated on the use of communication links while swarm flight of spacecraft may involve relative sensing [48], [49], [50] such as range and relative bearing measurements in 3-D. In fact, the use of relative sensing can further reduce the estimation errors of the distributed sensing algorithms that would otherwise use only GPS and intersatellite crosslink.

\section{SPARse Aperture ApPlication AND PERFormance-Cost ANALYSis}

A key application of swarms of spacecraft is sparse aperture sensing or stellar interferometry. The swarm dynamics modelling and swarm GN\&C technologies described in Section III would enable swarm keeping and reconfiguration of a large number of apertures in LEO to realize synthetic aperture arrays comprised of femtosats. For example, we can find a transformation between the 3-D PRO and a circular projected plane that is normal to the target line-of-sight vector. For 


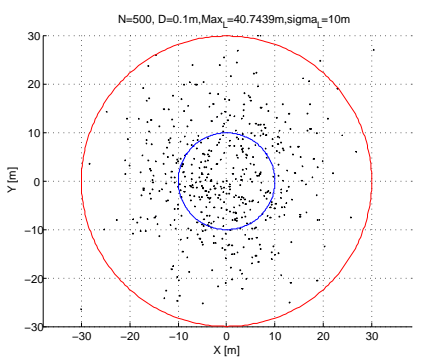

(a) Gaussian Random, $\sigma=10 \mathrm{~m}$ (blue), $3 \sigma=30 \mathrm{~m}$ (red)

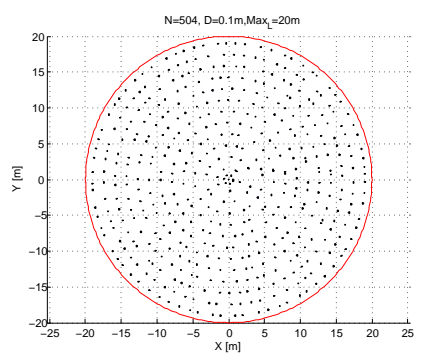

(c) Structured Disk, $\mathrm{L}=20 \mathrm{~m}$

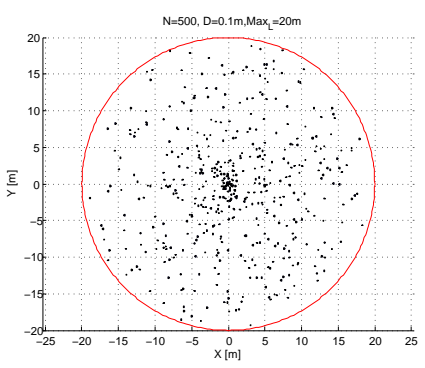

(e) Uniform Disk, $\mathrm{L}=20 \mathrm{~m}$

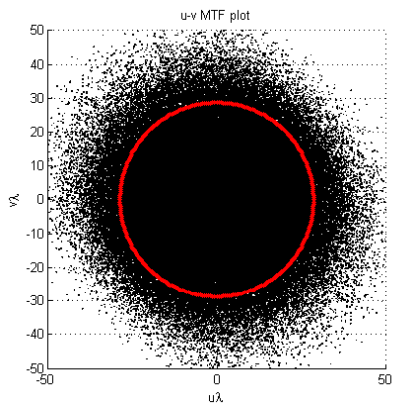

(b) MTF of (a), $D_{\text {eff }}=28 \mathrm{~m}$

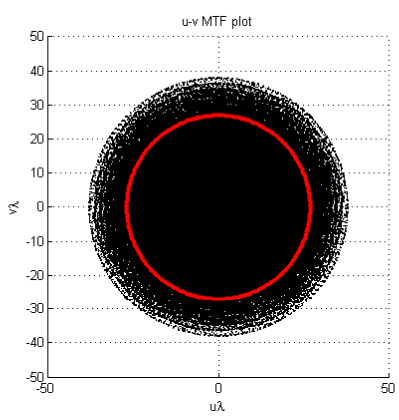

(d) MTF of (c), $D_{\text {eff }}=27 \mathrm{~m}$

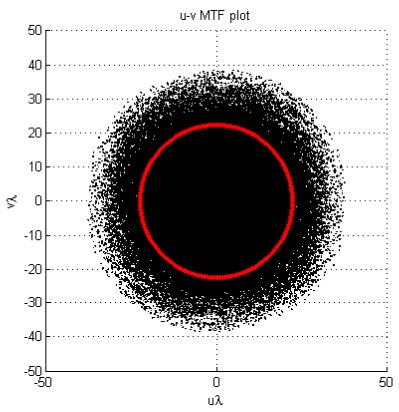

(f) MTF of (e), $D_{\text {eff }}=21 \mathrm{~m}$

Fig. 8. Random sparse array configurations (a,c,e) and their MTF plots (b,d,f), with 500 spacecraft. The diameter of each aperture is $10 \mathrm{~cm}$, while the effective diameter $\left(D_{\text {eff }}\right)$ for instantaneous u-v filling is given in $(\mathrm{b}, \mathrm{d}, \mathrm{f})$.

observing Earth's surface, $z_{\max }$ in the LVLH frame can be set equal to the semi-major axis of the PRO projected in the $x-y$ plane so that a circular projected orbit (PCO) can be obtained in the $\mathrm{y}-\mathrm{z}$ plane. Enabled by the GN\&C strategies described in Section III, we introduce new swarm configuration arrays that can be constructed on projected circular relative orbits. By employing the interferometric imaging metrics, we also present a unique performance and cost analysis that illustrates the cost benefits of the femtosat swarm architecture. A comparative configuration-cost analysis is presented next.

\section{A. Random Sparse Aperture Arrays and Performance Analysis}

We can spread out radar or optical telescopes with an aim to achieve a resolution that is comparable to a large monolithic aperture. Because of the stringent requirement on interferometric beam combination [51], that is, the beams should be combined within a fraction of wavelength, telescopes for longer wavelengths, such as radio or submillimeter

wavelengths, appear to be a more promising application of sparse aperture sensing for the SWIFT swarm.

Three possible swarm configurations are considered depending on the distribution characteristic of femtosats, as shown in Fig. 8. Previously, random arrays have been studied for communication relay [52], antennas [53], and space-based radars [54]. All the configurations are assumed to be on a projected circular plane. As discussed in Section III, the passive relative orbits (PROs) of femtosats in the relative orbital frame can be of any arbitrary orientation such that the projected plane is always circular and normal to the line of sight (see Fig. 1b).

In a Gaussian Random Array shown in Fig. 8a , the locations of femtosats on the projected circular plane follow the Gaussian distribution with a variance $\sigma^{2}$. On the other hand, each spacecraft in a Structured Disk Array is distributed with a prescribed radial and angular separation, thereby ensuring a certain separation distance between spacecraft. This can be interpreted in the sense of the discrete uniform distribution. This array can also be randomly perturbed slightly due to sensor and control errors of each femtosat, as shown in Fig. 8c. The third configuration, shown in Fig. 8e, is called a Uniform Disk Array, since femtosats are spread by the continuous uniform distribution. In contrast with the Gaussian Random Array, we can prescribe the maximum radius of the array for both Structured and Uniform Disk Arrays.

The angular resolution is determined by the Rayleigh's criterion $\theta_{r}=1.22 \lambda / D$. For sparse aperture formations, the diameter $D$ of a monolithic aperture for the wavelength $\lambda$ of interest should be replaced by the effective diameter $D_{\text {eff }}$. Then, the central question is how to determine the effective diameter of the random formation configurations (e.g., see Fig. 8). When looking at extended objects such as Earth's surface or faint distant nebulae, evaluation of an optical or radar system involves more than simply looking at a point source response from the point spread function (PSF), which determines the angular resolution by the method of full width at half maximum. However, the angular resolution alone is inadequate for many sparse-aperture or interferometric array applications. As elucidated in [51], a better metric to determine $D_{\text {eff }}$ is the modulation transfer function (MTF) that evaluates the contrast (modulation) transfer characteristic of snapshot imaging of extended objects. ${ }^{2}$ A 2-D projection of the MTF plot, called u-v points, can be determined by taking the autocorrelation points as follows

$$
u= \pm\left(x_{i}-x_{j}\right) / \lambda, \quad v= \pm\left(y_{i}-y_{j}\right) / \lambda
$$

where $\left(x_{i}, y_{i}\right)$ and $\left(x_{j}, y_{j}\right)$ are any possible pair of points within apertures, and $\lambda$ is the wavelength of interest.

In order to properly compare with monolithic filled apertures, we choose to define the effective diameter as the maximum radius of the MTF plots without singular (zero) uv points. The $D_{\text {eff }}$ of each configuration is denoted by the red circles in Fig. 8(b,d,f). We assume that each femtosat

\footnotetext{
${ }^{2}$ The PSF is the squared modulus of the Fourier transform of the complex pupil function. The optical transform function (OTF) is a Fourier transform of the PSF, and the MTF is an absolute value of the OTF.
} 
carries a radar aperture of $10 \mathrm{~cm}$ in diameter. By determining the array size that yields an instantaneous filled $u-v$ coverage in the MTF with 500 apertures, the effective diameters for each configuration are computed as $28 \mathrm{~m}, 27 \mathrm{~m}$, and 21 $\mathrm{m}$, respectively. Hence, the Gaussian Random and Structured Disk Arrays can achieve a finer angular resolution for an instantaneous full u-v coverage.

This result does not imply that a sparse aperture array with 500 femtosats can only achieve the effective diameter of $28 \mathrm{~m}$. If we can integrate images for a longer period of time, similar to Synthetic Aperture Radar (SAR) and Very Large Baseline Interferometry (VLBI), the finest angular resolution achieved by the array is determined by the largest separation distance between the apertures. In other words, $D_{\text {eff }}$ becomes the max separation distance. For example, a swarm of femtosats can be spread over a distance of 1-5 km, thereby yielding a much finer angular resolution. This is technologically feasible since the apertures distributed on the PROs will be rotating with respect to the center of the relative frame, as discussed in Section III. Such a large separation distance can be beneficial for longer wavelengths, since the angular resolution is inversely proportional to the wavelength. However, such a large baseline length will inevitably lead to much sparser u-v filling with many singular points (zero contrast), thereby decreasing the sensitivity or signal-to-noise ratio (SNR). Also, a noncompact $\mathrm{u}-\mathrm{v}$ coverage cannot be used for snapshot imaging. Hence, the array design of sparse apertures is a trade-off between the angular resolution of a point target and the sensitivity or contrast characteristics of a filled aperture. In order to properly compare with a fully-filled monolithic aperture, in particular for the cost analysis in Section IV-C, we proceed to use an instantaneous $\mathrm{u}-\mathrm{v}$ coverage.

\section{B. Novel Swarm Golay Arrays and Performance Analysis}

By examining the u-v coverage on the MTF plots, we can find that there are many redundant $\mathrm{u}-\mathrm{v}$ points that could have been saved to increase the effective diameter. In this paper, we introduce new random sparse arrays that can further optimize the number of spacecraft needed for a target effective diameter as shown in Fig. 9. Let us recall an optimal imaging configuration designed for a small number of apertures $(\mathrm{N}=3-12)$, proposed by Golay [55]. These arrays are all nonredundant and optimized for compactness in the u-v plot. Since we can construct a filled $\mathrm{u}-\mathrm{v}$ coverage by using the proposed random arrays shown in Fig. 8, a nice extension of Golay arrays is to spread multiple femtosats within the fractionated, virtual aperture diameters defined by the original Golay configuration. The proposed Swarm Golay-6, Swarm Golay-9, and Swarm Golay-12 are shown in Fig. 9. The MTF characteristic of a Swarm Golay-3 Array turns out to be similar to that of a Gaussian Random or Structured Disk Array, hence the Golay-3 is omitted here. By computing the MTF without discontinuous singular u-v points, the effective diameter $D_{\text {eff }}$ is determined as shown in Fig. 9(b,d,f). By using the same number of femtosats $(\mathrm{N}=500)$, the Swarm Golay-9 or Golay-12 Arrays achieve much larger effective diameters. Hence, we can further reduce the system mass or cost by utilizing Swarm Golay Arrays.

The results from Figs. 8 and 9 are summarized in Fig. 10

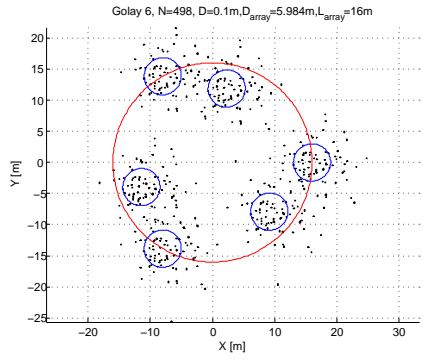

(a) Swarm Golay-6, L $=16 \mathrm{~m}$, $\mathrm{D}_{\text {Golay }}=5.98 \mathrm{~m}$

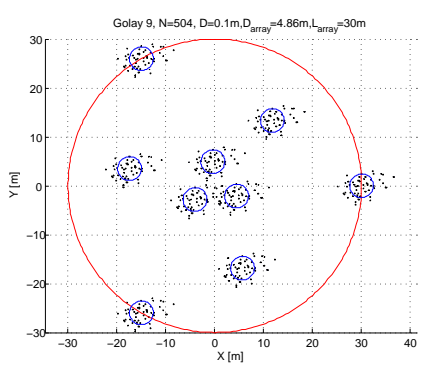

(c) Swarm Golay-9, L $=30 \mathrm{~m}$, $\mathrm{D}_{\text {Golay }}=4.86 \mathrm{~m}$

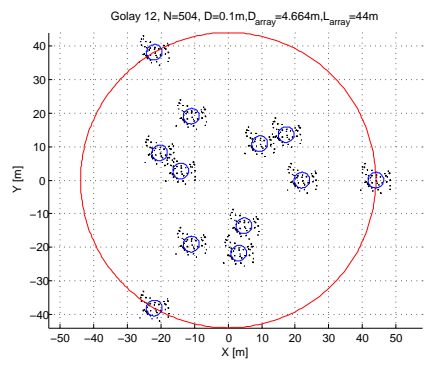

(e) Swarm Golay-12, L $=44 \mathrm{~m}$, $\mathrm{D}_{\text {Golay }}=4.66 \mathrm{~m}$

Fig. 9. Swarm Golay configurations $(\mathrm{a}, \mathrm{c}, \mathrm{e})$ and their MTF plots $(\mathrm{b}, \mathrm{d}, \mathrm{f})$ for 500 spacecraft with the aperture diameter of $10 \mathrm{~cm}$. The effective diameters $\left(D_{\text {eff }}\right)$ for instantaneous u-v filling are larger than those in Fig. 8.

for various aperture sizes and numbers. In general, the results are in excellent agreement with prior work [53] indicating that a smaller number of apertures are needed in random arrays. What is new here is that we can further reduce the number of apertures required to achieve a comparable aperture performance (e.g., angular resolution) by employing Swarm Golay configurations. As shown in Fig. 10a, we can more dramatically improve the angular resolution of the swarm aperture arrays by increasing the number of spacecraft. This result can be viewed as a compelling rationale behind the swarm architecture that employs thousands of femtosats.

For the same number of femtosats, the Golay-12 Array achieved a larger effective diameter, followed by the Golay9, the Golay-6, and the Gaussian Random Array. Other information we can extract here is the size of the array needed to achieve the desired $D_{\text {eff }}$ based on the full u-v coverage requirement. For example, the Gaussian Random Array needs the largest array size, hence possibly imposing more demanding communication requirements. As discussed in Section II-B, the long-distance communication subsystem can drive the system mass and cost. However, in the Swarm 


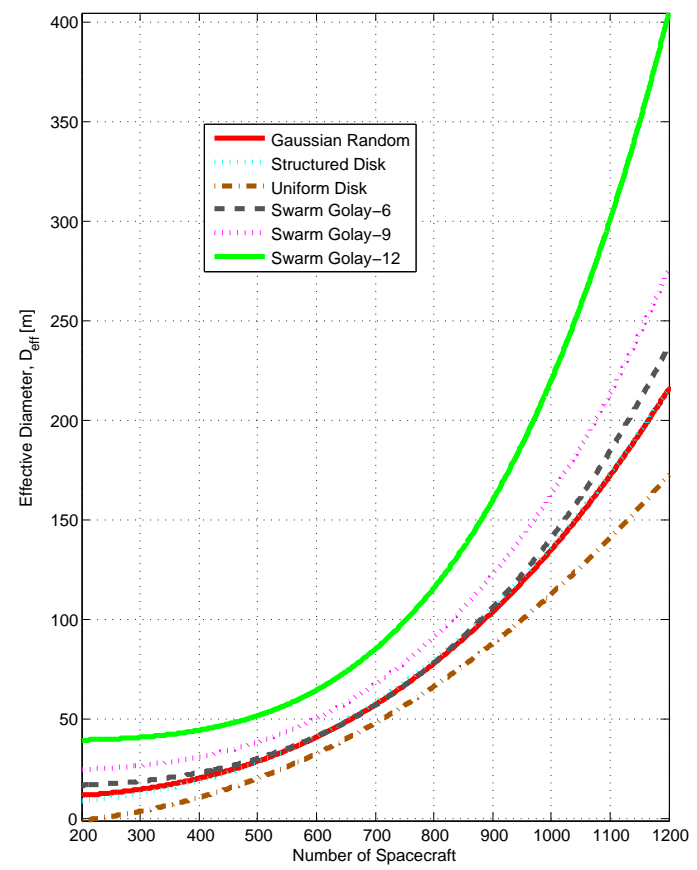

(a) Effective diameter as a function of number of apertures

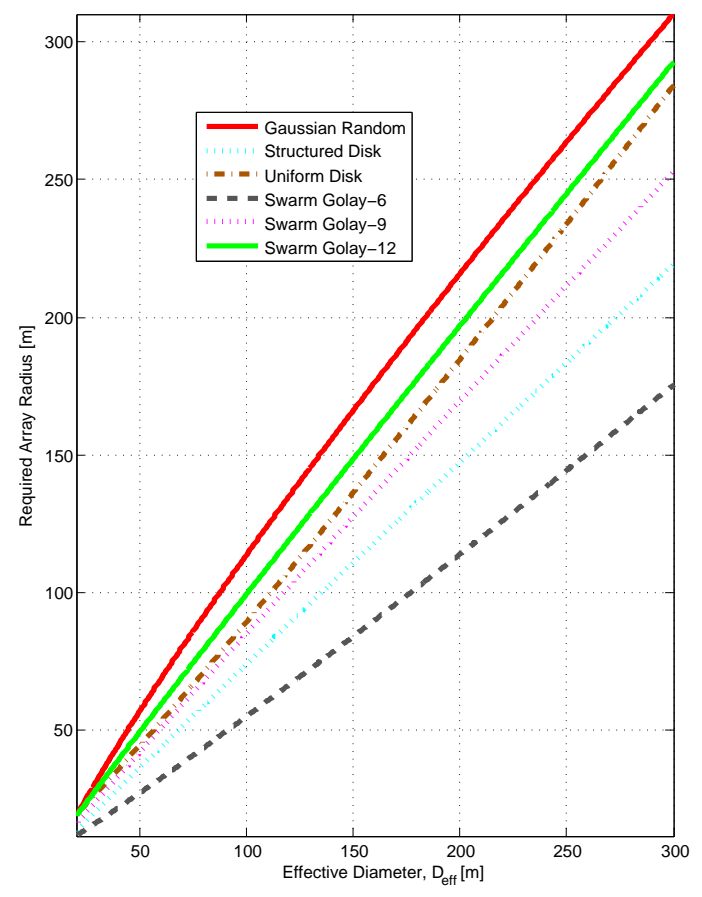

(b) Array size as a function of effective diameter

Fig. 10. Effective diameters from swarm aperture configurations

Golay Arrays, a swarm of femtosats can be divided to 6, 9, or 12 subset groups. As a result, such fractionated grouping can be advantageous in swarm controls and communications.

\section{System Cost Analysis}

We can hypothesize from Fig. 10 that the 400-m effective diameter constructed from 1,200 femtosats can be manufactured at a fraction of the manufacturing cost of an immense monolithic spacecraft carrying a 400-m diameter telescope that cannot be launched and built with the currently existing technology. In this section, we present an important cost analysis corroborating this dramatic cost saving of the spacecraft swarm architecture.

In order to compare the system cost of building and launching a monolithic aperture with that of sparse aperture arrays presented in the previous section, we modify the NASA Advanced Mission Cost Model [56] by multiplying it with $1 / \lambda^{-0.5}$

$$
\begin{aligned}
\text { cost }= & \$ 2.25 \text { billion } \times(\text { mass } / 10,000 \mathrm{~kg})^{0.654} \\
& \times\left(1.555^{\text {difficulty level }}\right) \times\left(N^{-0.406}\right) \times 1 / \sqrt{\lambda}
\end{aligned}
$$

where $N$ is the number of flight systems; and difficulty levels are: $-2=$ very low, $-1=$ low, $0=$ average, $1=$ high, and $2=$ very high.

The cost model given in Equation (9) computes the cost of a $1-\mathrm{kg}$ CubeSat to be $\$ 2$ million, which is close to the maximum launch cost of a CubeSat. This model also correctly predicts the project cost of infrared (IR) space telescopes such as the Hubble Space Telescope (HST) and the James Webb Space Telescope (JWST) to be about $\$ 4$ billion by substituting the mass and the difficulty level ( 2 for JWST and 1 for HST). However, how can we predict a cost for a large monolithic spaceborne radio telescope? Here, we consider the impact of Wavelength of Diffraction-Limited Performance (WDLP) by adding a factor of $1 / \lambda^{-0.5}$ where $\lambda$ is in $\mu \mathrm{m}$. The additional factor $\lambda^{-0.5}$, proposed by Stahl [57], match the existing cost data of ground based telescopes well. In a nutshell, this additional factor indicates that a radar space telescope with the wavelength $1 \mathrm{~m}$ is 1,000 times less expensive than a telescope of $1 \mu \mathrm{m}$ IR wavelength.

The project cost of a large monolithic space radar can be predicted, as shown in Fig. 11, to be a function of the diameter by assuming that the mass of the telescope is proportional to $D^{2}$. Consequently, the cost of a large monolithic space-based radar is proportional to $D^{1.308}$. The exponent of 1.308 agrees relatively well with the exponent 1.12 of the space telescope parametric cost model by Stahl [56]. Note that this number is smaller by a factor of two than the popular Meinel's cost model $D^{2.7}$ for ground telescopes, which includes the cost of telescope mount and dome. The key point here is not the exact amount of the predicted cost, but the exponent of the diameter that indicates a cost trend as a function of the diameter size. By identifying such a Cost Estimation Relationship (CER), we can determine significant cost drivers in the design process.

Similarly, we can compute the cost of building a 100-gram femtosat from (9). The total cost of a swarm array can be computed by multiplying the number of femtosats required in Fig. 10a for a given effective diameter. The result is shown in Fig. 11. As summarized in Table III, we can conclude that the cost exponent of the monolithic aperture is much steeper than that of the proposed swarm array configurations. As a result, we can dramatically save the system cost of launching a large radar aperture by deploying swarms of much smaller apertures. If the cost of fabricating a single femtosat is higher than expected, the results shown in Fig. 11 still indicate that there exists a break-even point between a monolithic telescope and a swarm array. In other words, in order to take advantage of the cost savings from the swarm, we need to increase the number of apertures or femtosats. This conclusion justifies 


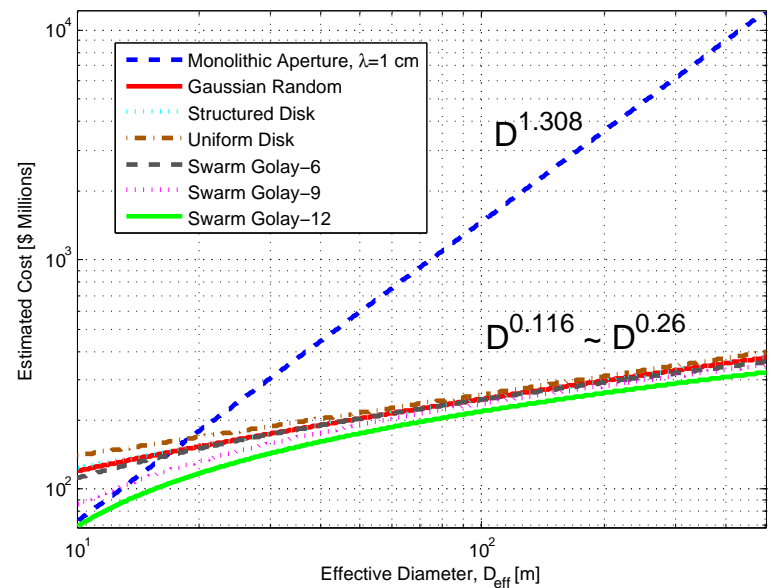

Fig. 11. The system cost analysis shows that the swarm configurations, defined in Figs. 8 and 9, are much more cost-effective than a monolithic telescope even without considering the learning curve saving.

TABLE III

Comparison of COST AS A FUnCtion of $D$ OR $D_{\text {EFF }}$

\begin{tabular}{|c|c|c|c|c|c|}
\hline Meine & Monolith & Gaussian Structur & 1 Uniforr & Swarm & Swarm Swarm \\
\hline Law & Aperture & Random Disk & Disk & Golay-6 & Golay-9 Golay-12 \\
\hline$D^{2.7}$ & $D^{1.308}$ & $D_{\mathrm{eff}}^{0.217} \quad D_{\mathrm{eff}}^{0.236}$ & $D_{\text {eff }}^{0.26}$ & $D_{\text {eff }}^{0.157}$ & $D_{\mathrm{eff}}^{0.132} D_{\mathrm{eff}}^{0.116}$ \\
\hline
\end{tabular}

the rationale behind a large number of femtosats. Also, it is expected that the benefits from the swarm architecture is more substantial when we factor in the learning cost saving as well as risk-reduction effects that are not captured in this cost analysis.

\section{CONCLUSION}

The proposed SWIFT project aims to develop hundreds or thousands of small femtosatellites and establish effective swarm GN\&C strategies for potential synthetic aperture applications. Based on the functional requirements of components, we presented functional baseline femtosat designs. The successful development of a fully capable 100-gram-class femtosat hinges on successful miniaturization of (a) propulsion systems; (b) component multifunctionality or multichip modules; and (c) low-mass, low-power electronics for longdistance communication. We also introduced new random sparse aperture arrays that can further optimize the number of spacecraft needed for a target effective diameter for an instantaneous imaging purpose. By using the same number of femtosats, the proposed Swarm Golay Arrays were shown to achieve much larger effective diameters, thereby implying that we could significantly reduce the system mass or cost by utilizing Swarm Golay Arrays. The cost analysis presented in this paper provides a compelling rationale behind the swarm architecture employing thousands of femtosats. The number of femtosats (100s-1000s) along with their modest sensing, control, and communication capabilities present new GN\&C challenges that have not been addressed in previous studies of formation flying. We explored potential solutions to the dynamic modeling and GN\&C challenges associated with femtosat swarms. The femtosat swarm architecture builds on a variety of distributed space systems by further maximizing the degree of distribution at a level of what is technologically feasible. Consequently, the enabling GN\&C technologies derived from the femtosat swarm would advance the state of the art in distributed sensing and control of multiagent systems controlled in 3-D. Furthermore, the successful implementation of the femtosat swarm architecture would result in a transformative innovation in space systems design, which has been dominated by monolithic spacecraft systems.

\section{ACKNOWLEDGEMENTS}

The research was carried out in part at the Jet Propulsion Laboratory, California Institute of Technology, under a contract with the National Aeronautics and Space Administration. (c) 2013 California Institute of Technology. Government sponsorship acknowledged. The SWIFT Swarm Project team includes David Bayard, Ed Mettler, Behchet Acikmese, Lars Blackmore, Milan Mandic, Marco Quadrelli, Risaku Toda, Eduardo Urgiles, Thor Wilson, Mohammad Mojarradi, Don Hunter, Ryan Kinnet, Dhak Muthulingam, Colleen MaresseReading, Sohrab Mobasser, Charles Bergh, John Zimer at JPL; Daniel Morgan at UIUC; and Raman Mehra at Scientific Systems Company, Inc.

\section{REFERENCES}

[1] O. Brown, P. Eremenko, and P. D. Collopy, "Value-centric design methodologies for fractionated spacecraft: Progress summary from phase 1 of the darpa system F6 program," in AIAA SPACE Conf. \& Exposition, Pasadena, CA, 2009, AIAA 2009-6540.

[2] I. Nason, J. Puig-Suari, and R. Twiggs, "Development of a family of picosatellite deployers based on the CubeSat standard," in IEEE Aerospace Conf., vol. 1, BigSky, MT, 2002, pp. 457-464.

[3] J. A. Atchisona and M. A. Peck, "A passive, sun-pointing, millimeterscale solar sail," Acta Astronautica, vol. 67, pp. 108-121, Jul.-Aug. 2010.

[4] D. J. Barnhart, T. Vladimirovaa, A. M. Bakerb, and M. N. Sweetin, "A low-cost femtosatellite to enable distributed space missions," Acta Astronautica, vol. 64, pp. 1123-1143, Jun.-Jul. 2009.

[5] F. Y. Hadaegh, B. Acikmese, D. S. Bayard, G. Singh, M. Mandic, S.J. Chung, and D. Morgan, "Guidance and control of formation flying spacecraft: From two to thousands," Adventures on the Interface of Mechanics and Control, Edited by K. Alfriend, et al., pp. 327-371, 2012, Tech Science Press.

[6] M. Martin, S. Kilberg, and J. Winter, "Techsat 21 and revolutionizing space missions using microsatellites," in 15th AIAA/USU Conf. Small Satellites, Logan, Utah, Aug. 2001.

[7] D. P. Scharf, F. Y. Hadaegh, and S. R. Ploen, "A survey of spacecraft formation flying guidance and control (Part I): Guidance," in American Control Conf., Denver, CO, June 2003, pp. 1733-1739.

[8] _ - "A survey of spacecraft formation flying guidance and control (Part II): Control," in American Control Conf., Boston, MA, Jun. 2004, pp. 2976-2984.

[9] D. P. Scharf, J. A. Keim, and F. Y. Hadaegh, "Flight-like ground demonstrations of precision maneuvers for spacecraft formations - Part I.” IEEE Systems J., vol. 4, pp. 84-95, 2010.

[10] — "Flight-like ground demonstrations of precision maneuvers for spacecraft formations- Part II.” IEEE Systems J., vol. 4, pp. 96-106, 2010.

[11] J. R. Wertz and W. J. Larson, Space Mission Analysis and Design, 3rd ed. El Segundo, CA: Microcosm Press, 1999.

[12] G. E. Moore, "Cramming more components onto integrated circuits," Electronics, vol. 38, no. 8, Apr. 1965.

[13] Panasonic Corp., "MIPTEC 3-D packaging technology," http://www3.panasonic.biz/ac/e/tech/miptec/index.jsp, 2012.

[14] C. Lee, S. Y. Bae, S. Mobasser, and H. Manohara, "A novel silicon nanotips antireflection surface for the micro sun sensor," Nano Lett., vol. 5, no. 12, pp. 2438-2442, 2005.

[15] Invensense Inc., "Six-axis (gyro + accelerometer) MEMS motiontracking ${ }^{T M}$ devices, http://www.invensense.com/mems/gyro/ sixaxis.html, 2013." 
[16] Analog Devices Inc., "MEMS inertial measurement units," http://www.analog.com/en/mems-sensors/mems-inertial-measurementunits/products/index.html, 2013.

[17] D. Platt, "A monopropellant milli-newton thruster system for attitude control of nanosatellites," in Proc. 16th Annual USU Conference on Small Satellites, 2002, SSC02-VII-4.

[18] D. Lewis, S. Janson, and R. Cohen, "Digital micro-propulsion project," Sensors and Actuators A, Physical, vol. 80, no. 2, pp. 143-154, 2000.

[19] D. Morgan, S.-J. Chung, L. Blackmore, B. Acikmese, D. Bayard, and F. Y. Hadaegh, "Swarm-keeping strategies for spacecraft under $J_{2}$ and atmospheric drag perturbations," J. Guid., Control, Dyn., vol. 35, no. 5, pp. 1492-1506, 2012.

[20] K. T. Afriend, S. R. Vadali, and H. Schaub, "Formation flying satellites: Control by an astrodynamicist," Celestial Mechanics and Dynamical Astronomy, vol. 81, pp. 57-62, 2001.

[21] I. Chang, S.-J. Chung, and L. Blackmore, "Cooperative control with adaptive graph Laplacians for spacecraft formation flying," in IEEE Conf. Decision and Control, Atlanta, GA, Dec. 2010, pp. 4926-4933.

[22] G. Xu and D. Wang, "Nonlinear dynamic equations of satellite relative motion around an oblate Earth," J. Guid., Control, Dyn., vol. 31, no. 5, pp. $1521-1524,2008$

[23] S. D'Amico and O. Montenbruck, "Proximity operations of formationflying spacecraft using an eccentricity/inclination vector separation," $J$. Guid., Control, Dyn., vol. 29, no. 3, pp. 554-563, 2006.

[24] T. A. Lovell and S. G. Tragesser, "Guidance for relative motion of low earth orbit spacecraft based on relative orbit elements," in AIAA/AAS Astrodynamics Specialist Conf., Providence, RI, Aug. 2004, AIAA 2004-4988.

[25] H. Schaub, S. R. Vadali, J. L. Junkins, and K. T. Alfriend, "Spacecraft formation flying control using mean orbit elements," J. Astronautical Sciences, vol. 48, no. 1, pp. 69-87, Jan.-Mar. 2000.

[26] R. M. Murray, "Recent research in cooperative control of multivehicle systems," J. Dyn. Syst., Meas., Control, vol. 129, pp. 571-583, 2007.

[27] J. S. Shamma, Ed., Cooperative Control of Distributed Multi-Agent Systems. West Sussex, England: John Wiley \& Sons, Ltd, 2007.

[28] S.-J. Chung and J.-J. E. Slotine, "Cooperative robot control and concurrent synchronization of lagrangian systems," IEEE Trans. Robot, vol. 25, no. 3, pp. 686-700, 2009.

[29] S.-J. Chung, U. Ahsun, and J.-J. Slotine, "Application of synchronization to formation flying spacecraft: Lagrangian approach," J. Guid., Control, Dyn., vol. 32, no. 2, pp. 512-526, 2009.

[30] P. K. C. Wang, F. Y. Hadaegh, and K. Lau, "Synchronized formation rotation and attitude control of multiple free-flying spacecraft," J. Guid., Control, Dyn., vol. 22, no. 1, pp. 28-35, 1999.

[31] S.-J. Chung and D. W. Miller, "Propellant-free control of tethered formation flight, part 1: linear control and experimentation," J. Guid., Control, Dyn., vol. 31, no. 3, pp. 571-584, May.-Jun. 2008.

[32] S.-J. Chung, J.-J. E. Slotine, and D. W. Miller, "Propellant-free control of tethered formation flight, part 2: nonlinear underactuated control," $J$. Guid., Control, Dyn., vol. 31, no. 5, pp. 1437-1446, Sep.-Oct. 2008.

[33] P. Tsiotras and J. Luo, "Control of underactuated spacecraft with bounded inputs," Automatica, vol. 36, no. 8, pp. 1153-1169, 2000.

[34] S.-J. Chung, S. Bandyopadhyay, I. Chang, and F. Y. Hadaegh, "Phase synchronization control of complex networks of Lagrangian systems on adaptive digraphs," Automatica, vol. 49, no. 5, pp. 1148-1161, 2013.

[35] P. Wang, F. Hadaegh, and K. Lau, "Synchronized formation rotation and attitude control of multi free-flying spacecraft," J. Guid., Control, Dyn., vol. 22, no. 1, pp. 28-35, 1999.

[36] R. W. Beard, J. Lawton, and F. Y. Hadaegh, "A coordination architecture for spacecraft formation control," IEEE Trans Control Syst. Technol., vol. 9, no. 6, pp. 777-790, 2001.

[37] Q.-C. Pham and J.-J. E. Slotine, "Stable concurrent synchronization in dynamic system networks," Neural Netw., vol. 20, no. 1, pp. 62-77, Jan. 2007.

[38] S.-J. Chung and M. Dorothy, "Neurobiologically inspired control of engineered flapping flight," J. Guid., Control, Dyn., vol. 33, no. 2, pp. 440-453, Mar.-Apr. 2010.

[39] S.-J. Chung and J.-J. E. Slotine, "On synchronization of coupled HopfKuramoto oscillators with phase delays," in 49th IEEE Conf. Decision and Control (CDC), Atlanta, GA, Dec. 2010, pp. 3181-3187.

[40] D. Morgan, S.-J. Chung, and F. Y. Hadaegh, "Spacecraft swarm guidance using a sequence of decentralized convex optimizations," in AIAA Astrodynamics Specialist Conf., Minneapolis, MN, Aug. 2012, AIAA 2012-4583.

[41] — - "Model predictive control of swarms of spacecraft using sequential convex programming," J. Guid., Control, Dyn., 2014, in press.
[42] M. G. Earl and R. D'Andrea, "Iterative MILP methods for vehicle control problems," IEEE Trans. Robot, vol. 21, no. 6, pp. 1158-1167, 2005.

[43] A. Richards, T. Schouwenaars, J. P. How, and E. Feron, "Spacecraft trajectory planning with avoidance constraints using mixed-integer linear programming," J. Guid., Control, Dyn., vol. 25, no. 4, pp. 755-764, 2002.

[44] S. Bandyopadhyay, S.-J. Chung, and F. Y. Hadaegh, "Probabilistic swarm guidance using inhomogeneous markov chains," IEEE Transactions on Control of Network Systems, 2014, in review.

[45] R. Olfati-Saber, "Kalman-consensus filtering: Optimality, stability, and performance," in 48th IEEE Conf. Decision, Control, Shanghai, China, Dec. 2009.

[46] S. Bandyopadhyay and S.-J. Chung, "Distributed estimation using bayesian consensus filtering," in American Control Conf., Portland, OR, Jun. 2014.

[47] J. L. Speyer, "Computation and transmission requirements for a decentralized linear-quadratic-gaussian control problem," IEEE Trans. Autom. Control, vol. 24, no. 2, pp. 266-269, Apr. 1979.

[48] R. S. Smith and F. Y. Hadaegh, "Control of deep-space formationflying spacecraft: Relative sensing and switched information," J. Guid., Control, Dyn., vol. 28, no. 1, pp. 106-114, Jan.-Feb. 2005.

[49] — - "Closed-loop dynamics of cooperative vehicle formations with parallel estimators and communication," IEEE Trans. Autom. Control, vol. 52, no. 8, pp. 1404-1414, 2007.

[50] — "Distributed estimation, communication and control for deep space formations," IET Control Theory Applications, vol. 1, no. 2, pp. 445451, Mar. 2007.

[51] S.-J. Chung, D. W. Miller, and O. L. de Weck, "ARGOS testbed: study of multidisciplinary challenges of future spaceborne interferometric arrays," Opt. Eng., vol. 43, pp. 2156-2167, Sep. 2004.

[52] J. B. Vespoli, F. Haber, R. Berkowitz, and D. Yavuz, "A self-organizing random array communication relay," IEEE Trans. Communications, vol. COM-31, no. 4, pp. 484-492, Apr. 1983.

[53] K. C. Kerby and J. T. Bernhard, "Sidelobe level and wideband behavior of arrays of random subarrays," IEEE Trans. Antennas Propagation, vol. 54, no. 8, pp. 2253-2262, Aug. 2006

[54] J. K. Schindler and H. Steyskal, "Sparse, random array processing for space-based radar," in 36th IEEE Southeastern Symposium on System Theory, Mar. 2004.

[55] M. Golay, "Point arrays having compact non-redundant autocorrelations," J. Optical Society America, vol. 61, pp. 272-273, 1971.

[56] H. P. Stahl, "Survey of cost models for space telescopes," Opt. Eng., vol. 49, no. 5, May 2010.

[57] H. P. Stahl, G. H. Rowell, G. Reese, and A. Byberg, "Multivariable parametric cost model for ground optical telescope assembly," Opt. Eng., vol. 44, no. 8, Aug. 2005. 